\title{
«Checks and Balances» au BMS
}

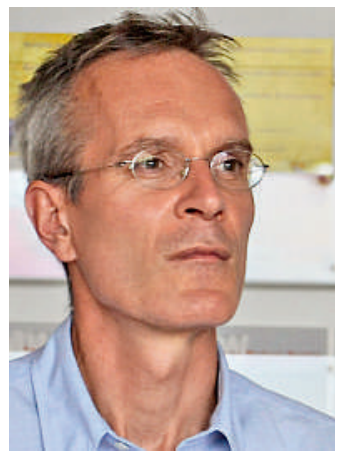

Bruno Kesseli
On ne risque pas de dévoiler un grand secret en disant que l'année qui s'achève a comporté d'importants défis pour la FMH. «DRG» et «Managed Care» sont deux mots clés suffisamment connus à cet égard.

L'objectif de cette chronique n'est pas de proposer une analyse de la manière dont la FMH et d'autres groupes au sein du corps médical ont fait face à ces enjeux politiques. Sur le plan rédactionnel, la question essentielle est plutôt de savoir comment le Bulletin des Médecins Suisses (BMS) a géré et gère encore les défis journalistiques liés à ces sujets et à d'autres, controversés au sein du corps médical.

Il est peut-être judicieux de rappeler, dans ce contexte, le «concept dual» - remarquable pour la revue d'une association - sur lequel se fonde le BMS depuis la création des Editions médicales suisses EMH en 1997 par les partenaires FMH et Verlag Schwabe. Outre sa fonction d'organe officiel chargé de communiquer de manière transparente les points de vue de la FMH aux membres et à un public plus vaste, intéressé par les questions de politique de la santé, le BMS a dès le départ été conçu comme une plateforme d'information et de discussion ouverte, destinée au corps médical et à tous les milieux en rapport avec le secteur de la santé. Même dans les périodes tendues, la FMH n'a jamais dévié de cette ligne de conduite et continue - avec, entre autres, deux représentants engagés au sein de la rédaction - à l'appliquer sans réserve, ce qui est tout à son honneur.

Le Bulletin des Médecins Suisses doit refléter ce qui préoccupe le corps médical, une devise que la rédaction se sent tenue de respecter. Il revient bien sûr à chaque lectrice, à chaque lecteur, de juger si et dans quelle mesure cela a été le cas durant l'année écoulée. De notre point de vue, le résultat est assez respectable. En ce qui concerne les sujets évoqués en préambule, je ne peux me souvenir d'aucun article ou courrier de lecteur rejeté, quel qu'ait été son degré de désaccord avec la "position officielle» de la FMH et ce, en dépit du fait que les pages rédactionnelles doivent être gérées de manière bien plus modeste qu'autrefois, pour des raisons d'économies.

Cela ne signifie pas pour autant que tout a toujours été rose, ni que tous les auteurs et groupements d'intérêts ont été satisfaits des processus journalistiques du BMS. Il serait d'ailleurs présomptueux de notre part de nous attendre à ce que tel soit le cas, alors que le fonctionnement du «modèle BMS» présuppose une collaboration équilibrée entre la rédac- tion et les différents organes de la FMH. «Checks and Balances» (poids et contrepoids) diraient les Américains.

Il va de soi que lorsque des textes en rapport avec la politique de la santé sont déposés, la FMH doit être impliquée dans son organe officiel et non les y découvrir avec surprise. Il ne s'agit pas d'une volonté de censure mais simplement d'un effort en vue de cultiver une communication cohérente et crédible de l'association, par exemple au travers d'un commentaire, lorsque celui-ci semble indiqué. Le fait que de tels processus soient chronophages dans un système de milice met parfois à rude épreuve les nerfs des auteurs, ainsi d'ailleurs que des collaborateurs du BMS, avant tout journalistes. Résoudre la quadrature du cercle dans ce domaine est à peu près aussi simple qu'en géométrie. Nous nous efforçons toutefois d'exploiter à fond le potentiel d'amélioration bien réel à notre disposition.

En attendant, certains signes réjouissants tendent à prouver que la FMH et la rédaction font plutôt du bon travail avec le BMS, de l'avis des principaux clients. On peut d'une part rappeler à ce sujet le sondage des lecteurs «medizinische Fachpresse» (presse médicale) réalisé l'an dernier par l'Institut für Marktund Sozialforschung gfs-zürich* et récompensé du label REMP**, qui a montré que le Bulletin des Médecins Suisses se plaçait non seulement loin devant la concurrence en termes de portée nationale, mais réalisait également d'excellents scores en matière de qualité. D'autre part, la décision prise en octobre de cette année par la Chambre médicale de soutenir les produits clés de la FMH - le BMS, le Forum Médical Suisse et Swiss Medical Weekly - au travers d'une contribution de base annuelle de 50 francs par membre, en raison du budget publicitaire en baisse de l'industrie pharmaceutique, peut être qualifiée de preuve de confiance. Même si le BMS reste essentiellement financé par les annonceurs, cette résolution montre que «sa voix» compte pour le corps médical.

Dans ces conditions, la rédaction du BMS peut envisager l'avenir avec un certain optimisme. Il va de soi que nous nous efforcerons, l'an prochain aussi, de répondre aux multiples attentes envers le BMS. Au nom de la rédaction, je vous souhaite, chères lectrices, chers lecteurs, de joyeuses fêtes et vous adresse tous mes vœux pour 2012.

Bruno Kesseli, rédacteur en chef \author{
sont scrupuleusement \\ vérifiés. \\ un organe central et neutre \\ d'instaurer la transparence \\ sur le marché des médias et \\ de la publicité. La recon \\ sance d'études de marché par \\ REMP est soumise à des
}

